From Individual Cognitive Maps to a Collective Cognitive Map: Prescriptive Guidelines and Measurement of Factors that Could Distort the Mapping Process

\author{
You Cheng ${ }^{1,2}$ and Elizabeth R. Chrastil ${ }^{1,2}$ \\ 1. University of California \\ Irvine \\ 2. University of California \\ Santa Barbara
}

\begin{abstract}
Author Note
You Cheng (D) https://orcid.org/0000-0002-3141-0104

Correspondence concerning this article should be addressed to You Cheng, Department of Cognitive Sciences, University of California, Irvine, Irvine CA 92617.
\end{abstract} E-mail: youc3@uci.edu 


\begin{abstract}
In the collective navigation scenario of a trio exploring in a foreign city, we propose a theoretical piece, which is a prescriptive guideline describing rational ways that can enable the trio to form a collective cognitive map. The guidelines center around three stages of exploration: the initial gathering of information, coming together to plan a route in the new city, and executing the exploration plan. Depending on the desires and goals of the group, they might explore together for some or all of the time, splitting up only when their individual goals diverge. The guidelines suggest an optimal plan for these different possibilities. We propose that a collective cognitive map is formed and improved during the entire cognitive navigation process as demonstrated by the trio drawing sketch maps, creating place maps, and revising other people's place maps. However, multiple factors could distort the navigation process at various points in the proposed prescriptive guidelines. These factors include individual differences (e.g., personal navigation ability, navigation anxiety, and sex), group dynamics (e.g., leaders and followers, group strategies), and the impact of the environment (e.g., language, culture, safety, and spatiality). We describe a thought experiment for testing collective navigation, including the measurement of these factors and the corresponding possible distortions in the collective map caused by these factors. Finally, we discuss future research directions, including using virtual environments and commercial applications. By utilizing our model, people can be flexible in resolving conflicting information during goal planning while still navigating efficiently.

Keywords: collective cognitive map, prescriptive guidelines, individual differences, social roles
\end{abstract}




\section{From Individual Cognitive Maps to a Collective Cognitive Map: Prescriptive Guidelines and Measurement of Factors that Could Distort the Mapping Process}

The minds of a trio exploring a foreign city are like three blank pieces of paper: as exploration begins, strokes and colors are gradually added to the paper, with occasional erases and redraws. The papers will turn into a useful cognitive map by the end of this exploration. Here, we provide a theoretical piece with prescriptive guidelines for the scenario, which aims to provide advice for this type of real-world situation. The guidelines could provide an assessment framework that could yield recommendations of how trios can most effectively navigate this challenge. In order to properly assess how this theoretical model best fits real data, we also discuss how different factors could lead to non-prescriptive behaviors and deviations from the prescriptive guidelines, as well as how researchers can measure those deviations.

\section{Prescriptive Guidelines}

\section{Information Collection - Drafting Individual Cognitive Maps}

We first assume that each person has a desire to plan the route, so then the rational way to allocate the task of cognitive map formation is to allow up to three different choices in route planning (i.e. each person will have his or her own route planning). Therefore, before making an exploration plan as a group, we will have the trio make a rational division of labor to collect information about the environment based off of their personal interests. For instance, the person who cares more about regional cuisine might talk to locals for advice, while the person who is more interested in places with art and history will likely look up information in a guidebook. The trio will not have access to any GPS navigation assistance, but they are allowed to read paper maps of the area. Depending on how each individual collect information, each person will either stay where he or she is or will move around but still keep close to the original position, staying within sight of other 
navigators. The primary goal of this process is to understand the layout of the region and choose potential destinations at an individual level. By the end, each tourist builds up a personalized cognitive map of the environment, which probably differs substantially from each other because they have different goals, routes taken, and strategies for acquiring the information.

These original individual cognitive maps will be tested by asking each member of the trio to complete three tasks - drawing sketch maps, creating place maps, and revising each other's place maps - sequentially and separately. Each person also needs to report whether they used a map during the information collection stage since exposure to maps might improve the precision of locating places and accuracy of the spatial layout in the sketch maps. Because the quality of sketch maps relies on an individual's navigation ability and even their drawing ability, other tests must also be conducted. A place map - created by adding place information to a base map containing only terrain and street information eliminates the process of putting different pieces together into a survey representation. Thus, it minimizes individual differences in the ability to integrate information as well as the influence of prior exposure to maps so that knowledge of place locations can be more directly evaluated. If the researchers plan to add any tests that require knowledge of location, distance, or direction, those tests need to be carried out before creating the place maps to minimize the influence of reading the base maps.

After creating place maps, each person will get a copy of the place maps created by the other two and will give feedback on them. The feedback on each item of place information will fall into three main categories: (i) Agree, a place is similarly located in both place maps, or only requires minor modifications to reach agreement; (ii) Uncertain, either the place is mentioned in both place maps but is only vaguely-represented in the cognitive map of the person making the judgment, or the place is not mentioned in the rater's place map, indicating that the rater cannot make a determination; and (iii) Disagree/revise, a place is very differently located on the two place maps. This place map 
comparison task evaluates the strength and similarity of place information between the members' cognitive maps, rather than accuracy.

Drawing individual sketch maps, creating place maps, and revising each other's place maps, may not be necessary in all situations for the trios to efficiently pool their knowledge, but it can be used to verify the prescriptive guidelines in an experimental setting. These sketch maps and place maps prior to group planning will be the first versions that researchers will collect; later versions will be compared with this first version to determine how much additional information has been added. They will also be compared with the ground truth to evaluate the accuracy of environmental representation formed during individual information collection.

\section{Communication and Planning - Synthesizing a Collective Cognitive Map}

After the information collection and drafting of individual cognitive maps, the trio meets up, exchanges information, and makes decisions. Starting from this stage, each person will wear a voice recorder so that their interpersonal communication could be recorded for later analysis. This time, the trio will be given a common reference map with terrain, street, and place information, so that the group can list all goals on the map without arguing about the locations. Once all goals are listed, the places and routes of common interest will first be chosen to delineate the main navigation route. The next step is to make fair decisions about personal preference and will generally have three possible outcomes:

(i) If everyone agrees to also visit the places of personal interest to the other two, the solution is to plan a route that incorporates all destinations. The collective cognitive map will be a route that sequentially connects all the destinations from the three individual maps, i.e. an overlay of the three cognitive maps. This is the simplest situation, but the trio may not have enough time to see everything.

(ii) If each member of the trio is willing to sacrifice a few personal-interest goals, the 
collective cognitive map will be less information-loaded compared to the first one. It will be a piecemeal collage of sections from each individual cognitive map. However, it might be advantageous to select this option rather than the first one because it provides more time to visit all common goals.

(iii) If the trio has mutually exclusive personal interests (i.e. everyone insists on personal interests without accepting others'), separate plans must be determined. In this case, first a main route of common interest will be determined, and then destinations for each individual will be listed and grouped in clusters on the reference map. Each cluster will include at least three locations of interest, which means at least one for each person. A location on the main common route that has a comparable geographic distance to each of the personal-interest destinations will be selected in each cluster (similar to Huff (1964)) as the hub for separation and meeting. After arriving at each hub, group members will start to explore the personal-interest places along their individual branch routes. After a set period of time, the trio returns to the hub and continues exploring the main route together. In this situation, everyone needs to generate a personalized map of the collective information. Common to all of these cognitive maps is the main route and mutual goals. The personalized portion of each cognitive map is the individual branch route and the personal-interest goals. Detailed information from the branch routes will be well-represented for the person who had a personal-interest goal in that branch, but the branch will only be vaguely-labeled in the other two's collective cognitive maps. For example, the other two travelers might only know that the third traveler went north down a particular street. The representation of all the branch routes in each person's collective cognitive map (represented by their revised sketch maps) is likely more distorted from the ground truth compared with the representation of main routes and mutual goals; less effort is typically expended in reaching an agreement on details for the individual branch routes than for the collective main route. This is the most complicated situation but provides opportunities to examine variables that are not testable in the other two situations, such as 
testing whether anxiety level when navigating alone is different from when navigating in a group in the same navigation scenario.

After coming up with a common navigation plan, the trio will subsequently make revisions on their own previous sketch maps, update their own place maps, and revise each other's revised place maps. All maps, including the common reference map, will be submitted to the experimenters and the trio will no longer have a chance to revise or look at them again when executing the plan. This second version of the sketch map and the place map will be compared with the first version to examine the effect of the group planning process on the environmental understanding of each individual. These maps will be compared with the ground truth to evaluate the accuracy of the newly-added information since the first version. The consistency between the first and second version of revising each other's place maps enables the researchers to evaluate information synthesis during the planning and discussion.

\section{Execution of the Exploration Plan - Utilizing the Collective Cognitive Map}

Efficient collective navigation tasks assume that everyone knows where and when to go next after they reach each place. In other words, they need good time-based and event-based prospective memory, as well as good episodic memory. Prospective memory is memory about the future. Time-based prospective memory is the ability to remember to execute an action based on a time-related cue, for example, setting off to a museum at 3 pm. Event-based prospective memory is the ability to remember to execute an action based on an event-related cue, for example, setting off to a museum after visiting a park. Episodic memory is the collection of personal past experiences that occurred at a specific time and place. This is especially true in situation (iii), in which the reunion of the trio at the return node requires both precise timekeeping and effective backtracking.

The advantage of collective navigation is similar to the Many-Eyes Effect (Elgar, 1989; Pulliam, 1973) from ecological studies of animals. In the Many-Eyes Effect, as the 
group number increases, the average time an individual spends watching out for predators decreases, while hit rates of predator detection increases at a group level. Similarly, in humans, especially when exploring a foreign city, navigation in groups will be more efficient than doing it individually. Attention to potential dangers and to the task of wayfinding decreases at an individual level, but safety and wayfinding increases at the group level. The enhancement of safety will potentially bring down spatial anxiety (Lawton, 1994). Thus, there will be more time and a better all-around mood for each person to enjoy the exploration. As the old saying goes, "two heads are better than one".

When the exploration ends, the trio will be asked to draw sketch maps, create place maps, and revise each other's place maps again separately. This third version of the sketch map and the place map will be compared with their second version of the sketch map and the place map to evaluate their learning process during exploration. Then, they will be compared with the ground truth to evaluate the accuracy of the newly-added details and information since the second version. The consistency between the second and third version of revising each other's place maps tasks enables the researchers to evaluate the information synthesis during the execution of the plan.

\section{Non-Prescriptive Factors and Measurements}

Multiple factors could influence collective navigation and make the real navigation pattern deviate from the prescriptive guidelines. In addition to the analysis of the three sketch maps mentioned above, different factors should also be measured during each stage of navigation as independent variables for navigation that could lead to deviations from the rational guidelines. We will describe and categorize these factors in order of the three different phases of navigation process: prior to the task, during the task, and after the task. 


\section{Prior to the Task}

\section{Personal Navigation Ability}

Personal navigation ability is not only a good predictor for navigation in the real world, it could also be a primary factor for predicting social roles and navigation efficiency in a group. For example, if everyone's navigation ability is similar, the trio may be more likely to split up the navigation responsibility. The division could be realized either by all three people navigating together with no particular person being the leader or by a dynamic leader-follower state in which the leadership position rotates among the three people. There could also be an imbalance in navigation ability. For example, one person is good at navigating while the other two are poor navigators. In this case, the trio will be more likely end up in a fixed leader-follower state, with the superior navigator as the leader. If two of the trio are both equally good navigators, then the whole group will still likely end up in either a fixed or dynamic leader-follower state with the third, poorer navigator always be the follower.

Navigation efficiency, indicated by the time it takes to successfully complete the whole task, could have four possible relationships with individual navigation ability: First, the group with higher average navigation ability could have better collective navigation performance. Second, the group with the highest similarity of navigation ability regardless of level - could have better collective navigation performance. Third, collective navigation performance is positively related to the navigation ability of only the best navigator in the group. Fourth, collective navigation performance is negatively related to the navigation ability of only the laggard in the group. Correlations between navigation performance and these four factors will be carried out in order to tell which one of the four hypotheses, if any, fits the prediction.

In addition, individual navigation strategy might also play a role in the group's performance. An egocentric strategy is based on a viewer-centered representation of the environment, such that spatial information is formed with respect to oneself. For instance, 
a person only takes fixed routes by remembering turn-by-turn directions. An allocentric strategy is based on survey knowledge of the environment, such that spatial information is formed independent of the observer's view. For instance, a person frequently takes shortcuts. Thus, applying allocentric strategies often makes navigation more efficient. If all groups managed to execute their plans, the trio whose members have congruent strategies is more likely to navigate efficiently than the ones with divergent strategies. A congruent allocentric strategy is likely to be more efficient than a congruent egocentric strategy. However, groups with congruent strategies may be more likely to get lost due to systematic biases, such that each member of the trio makes the same types of errors or misses the same type of information. Preference for egocentric or allocentric strategies will be tested either by self-report or by calculating the frequency of taking shortcuts in navigation tasks.

We will use questionnaires and navigation tasks to assess ability. Commonly used navigation ability questionnaires such as the Santa Barbara Sense of Direction Scale (Hegarty et al., 2002) or paper-and-pencil tests of perspective taking abilities such as the Money Road Map Test (Money \& Alexander, 1966; Money et al., 1965) only take about 3 minutes to complete on average. However, self-report measures of strategy are only weakly correlated with objective measures of strategy (Boone et al., 2018); the trio might not really know about their own navigation strategy. Participants will also complete navigation tasks in virtual environments on a computer. For example, the dual solution paradigm (Marchette et al., 2011; Weisberg \& Newcombe, 2016) is a test of navigational strategy preferences, such as route following or taking shortcuts. The maze learning test (Chrastil \& Warren, 2014) measures people's ability to learn the pathways between locations in a complicated maze environment. These tests take more time to conduct, approximately 30 50 minutes each. Thus, researchers should make careful decisions in task selection based on the total test time available and the priority of testing each particular ability. 


\section{General Navigation Anxiety Test}

As mentioned in the prescriptive guideline, due to the Many-Eyes-Effect, individual spatial anxiety level is predicted to decrease during collective navigation. In order to test that hypothesis, individual navigation anxiety levels need to be assessed before the task as a comparison baseline. Navigation anxiety levels could also predict social roles, route planning, and navigation behavior in collective navigation. People with the least spatial anxiety may be more confident in playing leading roles. People with higher spatial anxiety level might choose to visit places of interest on the main road, talk less during the exploration, or select safer places as landmarks, such as hospitals or police stations. The group with lower average spatial anxiety level will be more likely to plan to navigate in situation (iii) which requires each member of the trio to navigate alone during part of the trip. Spatial anxiety will be measured using a spatial anxiety questionnaire (Lawton, 1994).

It is worth noting that the spatial anxiety measured here is a general anxiety, which refers to anxiety as a personal characteristic. In contrast, state anxiety refers to anxiety in the situation, in this case collective navigation. To dissociate the two concepts in this

paper, we refer to the general version of spatial anxiety as "general navigation anxiety" and the in-state version of spatial anxiety as "state navigation anxiety". State navigation anxiety levels might differ from general navigation anxiety levels and will be estimated immediately after the trio gets to the last stop. We will discuss state navigation anxiety in more details in the "after the collective navigation" section.

\section{Navigation Experience}

Navigation experience here refers to a person's lifetime mobility, which is measured by calculating the number of locations someone has visited beyond one's day-to-day range. This indicator can help researchers determine whether a person might gain novel navigation practice by traveling to many locations. Navigation experience was found to be positively correlated with navigation performance in a virtual maze (Padilla et al., 2017). 
This factor is likely to play an even more important role in the current scenario because people gain navigation experience by traveling in novel places. In this collective navigation task, the individual with the most navigation experience is most likely to take a leading role. At a group level, the trio with greater average navigation experience may perform better in general than those with less navigation experience. Groups that plan to navigate in situation (iii) are more likely to have higher navigation experience on average, so that each member of the trio might be more confident in traveling alone. Navigation experience will be measured by using a modified mobility questionnaire, in which subjects are asked to indicate whether they had visited places on a list of local and national locations (Padilla et al., 2017).

\section{Sex EG Age}

Sex differences have been found frequently in navigation studies. Males generally have better performance (Astur et al., 1998; Moffat et al., 1998), use allocentric strategies

more frequently (Coluccia \& Louse, 2004, Lawton, 1994, Lawton \& Kallai, 2002), and have lower general navigation anxiety (Lawton, 1994) than females. In collective navigation, if there are both male and female members, it is possible that males will be more likely to take leading roles. Groups that decide to navigate in situation (iii) are more likely to have three male navigators, as males tend to have lower general navigation anxiety and thus will be more comfortable navigating alone. Another factor that might relate to sex differences in collective tasks is prosociality, which means taking actions that benefit others. Females tend to be more prosocial than males (Espinosa \& Kováŕík, 2015; Soutschek et al., 2017). In collective tasks that require communication and collaboration such as collective navigation, females may be more likely to play the role of facilitator for the group's decision-making process. When there are conflicts with other group members over goal selection, route planning, or strategy choice, females may be more likely to compromise so that the group can reach an agreement more smoothly. For this reason, a group with all 
females is also less likely to have mutually exclusive goal locations, and thus less likely to end up in situation (iii). The group with more females may find collective navigation more enjoyable and could lead to increased sharing of spatial information.

Age is another important demographic factor that could influence navigation performance. Older people tend to have decreased navigation performance because their hippocampal function deteriorates with aging (Lester et al., 2017). Older adults use more egocentric strategies, while young people tend to use more allocentric strategies in navigation and tend to be more efficient (M. A. Harris \& Wolbers, 2014; Wiener et al., 2013). If there are both old and young people in the group, it is possible that young people will be more likely to take leading roles. However, younger navigators could also defer to older navigators' leadership, no matter the age group. Therefore, each subject's sex and age information must be recorded before the task and be used as a factor to test the group's performance in collective navigation. In addition, researchers could compare the word frequency for each person giving directions during the navigation process to evaluate the influence of sex and age.

\section{Personality}

Prior studies have found that personality (including conscientiousness, intellect, emotional stability, and extraversion) is related to sense of direction (Condon et al., 2015). In navigation in a social situation like collective navigation, personality may even play a bigger role. For example, the person who has a higher extraversion score may be responsible for communicating with local people or become the communication coordinator within the group. The person with a higher emotional stability score may become the first to calm down and take a leading role in emotionally intense situations, such as getting lost.

We will test personality by using the Big Five personality traits scale (Goldberg, 1990). 


\section{Prospective Memory}

Most navigation tasks only test one goal location at a time and participants' only task is to reach the goal accurately and efficiently. In contrast, traveling in the real world requires reaching multiple goals sequentially. More importantly, each time that travelers reach a goal, they will spend time visiting the goal location rather than going to the next goal immediately. In order to execute the navigation task as planned, travelers need to have good prospective memory, and in particular time-based prospective memory. If all members of the trio have bad prospective memory, they may not notice that they spent too much time visiting a location, such that they might have to skip the remaining places or might not even have enough time to reach the final destination. If the trio cannot finish the trip in time, they will fail the collective navigation task, no matter their navigation performance. However, good collective navigation may not require everyone to have good prospective memory. Groups in situations (i) and (ii) can keep a good track of time as long as there is one timekeeper.

In situation (iii), everyone will have to keep their own track of time when navigating separately. If, unfortunately, any member of the trio loses track of time and spends too long on a branch route, the other two will have to either wait at the hub or look for the no-show. However, it is risky for the other two to look for the no-show because they do not know the detailed information of the route and they might get lost. Therefore, all members in situation (iii) will have to have good prospective memory, since one person with bad prospective memory could devastate the whole group.

Time-based prospective memory is usually tested using a dual task paradigm. In a dual task, the online task that is usually unrelated to memory but costs cognitive resources, for example, a lexical decision task to tell a word from non-word. The parallel offline task is a time estimation task such as to press a key every 2 minutes. Accurate time interval estimation and less frequent checking of the clock is associated with better time-based prospective memory. 


\section{During the Collective Navigation}

To minimize interference with the exploration process, information during the navigation period will be collected indirectly.

\section{Social Roles}

There could be different combinations of social roles in the collective navigation task as observed during the navigation process. The primary types of the combination of navigation social roles include a no-leader state and a leader-follower state. In the no-leader state, people navigate together all the time. A leader-follower state in the trio could be either two-leaders-one-follower state or one-leader-two-followers state. Each of the subtypes can be either dynamic or fixed. A fixed one-leader-two-followers state always has one particular person as the leader. A dynamic one-leader-two-followers state rotates leadership among the members of the trio. The two-leaders-one-follower states work similarly.

In addition to the leader-follower dynamic, there are other social roles that facilitate collective navigation. For example, a coordinator is responsible for facilitating communication within the group. A timekeeper is responsible for keeping track of time for the group travel as planned and reminding other members of the time when necessary. These additional social roles may be taken by more than one person in some groups or never exist in others. Some other social roles may only exist in particular environments. For example, when visiting a city with a high malaria rate, a mosquito watcher role will be created to prevent the group from getting close to danger. Researchers will determine the social roles by observation and the result will be checked against participants' self-reports after navigation. As mentioned before, participants' communication during the exploration will also be recorded to study the formation and validate the observation of social roles. For example, the one with the highest cardinal direction-related word frequency could be considered a leader, while the one with the highest non-cardinal direction-related word frequency could be considered a coordinator and the one who rarely talks is considered 
simply a follower.

The researchers will check whether the observed social roles match the predictions from the pre-navigation measurements mentioned above, such as whether the person with best personal navigation ability within a group really becomes the leader. The combination of social roles itself can also be an independent variable to provide answers for many questions: Do the groups in situation (iii) tend to be in either a no-leader situation or a dynamic one-leader-two-followers situation when navigating on common routes? For navigation situations (i) and (ii) outlined earlier, is there a particular combination of social roles that maximizes navigation efficiency for the trio? If not, are social roles that are different from the predictions based on age, sex, etc. less efficient than the ones that match the predictions? Which combination of social roles is associated with the highest level of spatial anxiety? If the trio navigates in a fixed leader-follower state, are leaders more anxious than followers or vice versa? Which combination of social roles is most enjoyable, and do leaders enjoy the tour more than the followers or vice versa?

A special case occurs when the trio gets lost. This situation may never happen to all trios, but if it happens, it will be very interesting to see how the trio figures the way out. Do they totally switch to another combination of social roles? If they persist with a fixed leader-follower state, for example, do they change the leader? Each person's role in solving problems in this situation will be quite revealing about the group dynamics. The group's navigation resilience will be measured by the speed with which the trio finds their way back to the correct route.

\section{Time in Route}

When navigating together, does each group member have a similar memory for every location they stopped at? Do they all have better memory for locations where they spent more time? Situation (iii) provides the opportunity to examine many questions: Does the navigation strategy change when the trio navigates individually? Does each 
navigator choose to walk farther but take less time to visit places of personal interest, or do they choose to walk less but take more time sightseeing? Do individuals walk faster or slower compared with walking in a group? Do individuals walk faster on the way to the personal interest places or faster on the way back to the hub? Does everyone get back to the hub on time? If one of the navigators is late for the meeting, how would that influence the remaining collective navigation in terms of social roles, personal interaction, or future plans? The trio's time distribution at each location and their route information will be measured by observation as well as by using a GPS tracking system (e.g. wearing GPS tracking watches).

\section{After the Collective Navigation}

Several cognitive tests and self-report questionnaires will be implemented after the collective navigation task to enhance our understanding of each person's navigation performance. Each member will be tested separately on the measures listed here.

\section{Cognitive Tests}

Knowledge of the Environment. Although the trio agrees on a common route during route planning stage, it does not mean each person completely replaced their own cognitive map with spatial information from the common map. Even if each individual consciously accepts the entire common map and they have the same collective cognitive map after route planning, the collective cognitive map could become distorted for each individual during the execution. Although each member of the trio will be asked to draw a sketch map and create a place map after their navigation, sketch maps and place maps still do not fully evaluate people's total knowledge of the environment. For example, a person might simply draw a node on the sketch map or place map to represent a museum, but that simple node misses vital facets of that person's knowledge, such as which side of the street the building is facing, the museum's distinguishing features, or the relative direction and distance between locations. Therefore, in order to better understand how collective 
these individual cognitive maps are, a thorough assessment of each person's environment knowledge is needed and must be carried out before creating place maps to minimize information gained by studying the base maps.

Several objective tests will be implemented to evaluate people's knowledge about the environment including the direction from the final destination to different landmarks, the direction and distance between different landmarks, and the precision of locating landmarks. Immediately after the collective navigation, each person will be asked to point from the final destination in the direction of other locations they passed during navigation. They will also be asked to estimate the distance and direction between two of those locations. Participants will also be given a set of pictures, including both landmarks or locations they saw during the tour and locations which they never saw, to test their recognition memory. People who passively followed the leader during the entire navigation experience are expected to only recognize locations, without having accurate distance or direction knowledge. People in situation (iii) may only be able to answer questions related to landmarks or locations on the common main routes and their individual branch routes, without having accurate knowledge about landmarks or locations related to other people's individual branch routes.

State Navigation Anxiety Test. As the reference for spatial anxiety during collective navigation, each person's state navigation anxiety level will be estimated immediately after the last stop. Due to the Many-Eyes Effect, state navigation anxiety levels are expected to be lower than general navigation anxiety levels, although they might increase if there is an interpersonal or communication conflict during the navigation task or if any of the non-prescriptive factors play a role. For example, the group with higher average state navigation anxiety might walk closer to each other and keep similar pace. There is no standard state navigation anxiety questionnaire, but the researchers will develop one based on Lawton's general anxiety questionnaire (Lawson \& Riecke, 2014) and the state scale of the State-Trait Anxiety Inventory (STAI) (Spielberger et al., 1983). 
Groups navigating in situation (iii) will need to have separate questions about the main routes and the personal branch routes because state navigation anxiety levels could differ when navigating separately compared with navigating together on the common routes.

\section{Self-Reports}

Strategy. When working in groups, the individuals might compromise on their personally-preferred strategy to go along with the group's preference. The group members might even have differing interpretations about what strategy the group is using. In order to measure people's knowledge about strategy use in collective navigation, each person will report their navigation strategy and the reason why they utilized that strategy. The researchers will compare the subjective reported strategy with their rationale for applying the strategy, the personally-preferred strategy measured in personal navigation ability tests (if available), as well as the objective observation. The objective observation can reveal strategies by analyzing if the paths taken between two consecutive goal locations are direct or not, or whether the group is using distal or proximal cues to locate themselves. Again, trios from situation (iii) will be asked to report strategies on their individual branch routes and the common main routes separately.

Assessment of Personal Performance. In collective navigation, it is important to assess people's conscious awareness about their social roles. If the group navigates efficiently, is each member content with his or her own performance? If the group navigates poorly, does each person blame his or her own performance, blame others, or blame the group as a whole? Each person will report the role he or she played in the collective navigation process, reflect on whether their own performance was satisfactory, and provide reasons for giving that assessment.

Assessment of Other Peoples' Performance. Do people have objective recognition of the roles other people played in the collective navigation task? To what extent do people think other's performance contributes to the group performance? Do people think others should take more responsibility for good or poor performance? Each 
person will report the role the other two navigators played in the collective navigation, the impression of their performance, and the reasons for having that impression.

The researchers will compare the self-evaluations, the evaluations of the other navigators, and the researchers' own observation of the trio to determine whether there are any biases, such as personality, spatial anxiety, or social role in the team.

Awareness of the Impact of Non-Prescriptive Factors. Does each group member have similar feeling about the group's performance? If there are any problems during collective navigation, does everyone notice the problem? If everyone notices the problem, are they all willing to fix it in the future and fix it in similar way? Each person will assess the group's performance, provide feedback on the places they feel were unsatisfactory, and provide suggestions to improve it. It will be interesting to see whether the trio hones in on the same non-prescriptive factors as the researchers, or they might even report some crucial factors that even the researchers did not notice.

Travel Experience Rating. Does the level of enjoyment of the tour a trade off with energy investments into the collective navigation? Does the person who navigates better also enjoy the tour better? Or are the two factors not actually related? Each person's enjoyment level will be easily evaluated with a 7-point Likert scale.

Additionally, experimenters will also analyze navigators' interpersonal communication recordings to evaluate the reliability of their self-reports. For example, the person who enjoys the traveling itself most may spend more time chatting about scenes rather than wayfinding.

\section{Other Factors.}

Language $\mathbb{E}$ Culture. When a language barrier exists, it will prevent tourists from communicating with local people even if the foreign city has a large population. Therefore, if one member of the group is the sole speaker of the local language, that navigator might be selected as the leader in spite of age, sex, previous navigation experience, or own willingness to guide. Also, people in the group who do not speak the 
language in the visiting city might have higher state navigation anxiety even if he or she has lower general navigation anxiety. In addition, a language barrier may influence the route planning stage of the whole group. If the group cares about whether every member has equal access to site resources (e.g., being able to read and communicate), they may choose to go to places that are easy for everyone to visit. For example, a group of English native speakers visits Tokyo but only one of them speaks Japanese. If the group plans on giving everyone equal access to the places they visit, they might choose to only visit famous places of interest in Tokyo where the tour guides speak English or all the buildings and displays have an English translation. On the other hand, if the whole group cares less about whether everyone can communicate, and only requires one person who can communicate with locals or read Japanese descriptions, then they might just plan the route as described in the prescriptive guidelines. The ones who do not speak the foreign language might unconsciously walk closer with each other to ensure that no one gets lost.

In general, the greater the similarity between the trio and the foreign city, the more likely they will be willing to take risks to visit more places with a larger range. In the extreme case when none of the trio speaks the foreign language, then they may not be able to visit places they really want to visit; instead, their travel goal will lean towards arriving at home safely with a limited exploration range.

It is also worth noting that certain cultures that encourage collaboration over independence may favor collective navigation. For example, Talhem and his colleagues found that Chinese people who live in the rice-growing southern China are more interdependent than people who live in the wheat-growing north (Talhelm et al., 2014). Therefore, people from southern China may tend to collaborate more in collective navigation than people from northern China.

Safety. Safety information about the environment itself can also influence the route planning and navigation process by modulating state navigation anxiety and attention. If the foreign city has a notorious region with a high violent crime rate, the trio 
might avoid going to that region even if there is a very famous place of interest. This tendency of avoiding dangerous places may get stronger when there are more female members in the trio. If the trio decides to visit the location with a high crime rate, they may walk closer together and pay more attention to each other during the navigation. Imagine the trio walking from Central Park to Brooklyn in New York City, they may pay more attention to navigation and stay closer together, as it tends to get less safe further south. A similar pattern may also apply to health factors. For example, if the trio is visiting a city in the southern part of Chihuahua, Mexico - an area with a high risk of malaria - they may try to avoid places with more mosquitos and remind each other to watch out for mosquitos during the whole trip. However, if they are visiting Mexico City - where malaria is rare - mosquitoes in the places of interest will be less of a concern for the group.

Spatiality. Spatiality - properties (e.g., layout) of the environment itself could also influence the collective navigation behavior. Cities which were built in grid-like patterns that align with cardinal direction referents (e.g., south-north and east-west) may unconsciously encourage people to use allocentric strategies, while cities that were built in a more unsystematic way may encourage people to rely more on local landmarks. In a previous online study, researchers found that regional differences may even overshadow sex differences in giving directions. People who live in the northeast or southern U.S. - with many old cities whose property boundaries were established in unsystematic ways - tended to refer to landmarks when giving route directions. In contrast, people who live in the west or Midwest of the United States - where land boundaries and roads were laid out in a more systematic way - used more cardinal directions as navigation referents (Lawton, 2001). Therefore, the environment itself may shape people's navigation behavior, even though it is a foreign environment. When studying how a trio explores a new city, it may be necessary to determine the spatiality of the city each of them grew up in, and then compare how their previous experience matches the spatial properties of the new city. If one grew up in a city with similar spatiality as the new city, the person maybe more confident and efficient 
in using their personal preferred strategy, and thus, take the leader role of the trio.

The size of the environment and the distance to goal locations also need to be large enough so that the trip can last long enough (at least 40 minutes) to test many of the hypotheses mentioned above. The design of the city layout should have a certain degree of complexity, such as a low viewshed potential — being surrounded by skyscrapers or having a crowded space with an obscured view. Diminished viewshed potential could potentially influence people's navigation anxiety. With sufficient complexity, the wayfinding task will not lead to a "floor effect" - everyone finds the task a piece of cake - which would reduce group differences in navigation.

\section{Future Directions}

\section{Virtual Environments}

Virtual reality enables researchers to "move" outdoor navigation environments into the lab. Virtual environments provide better control of external variables, and behaviors taking place in the environments are easier to measure. In completing tasks that require teamwork, in this case collective navigation, participants who are physically distant from each other can be "teleported" into the same virtual environment and navigate together. Multiple trios can navigate in the same environment simultaneously, which greatly facilitates data collection. In order to study individual differences, researchers can easily decide the ability level of the trio by pre-selecting individuals with corresponding characteristics based on pre-navigation measurements. However, people were found to explore fewer regions and communicate less in virtual environments than real ones (H. Harris et al., 2009), so testing social navigation in virtual environments may not totally replicate behaviors in the real world. 


\section{Commercial Applications}

This prescriptive framework for collective exploration of a new city can be tested in the real world. The information we get from assessments can further be applicable in real situations. For example, mobile food recommendation applications (e.g., Yelp) and real-world scavenger hunt game companies (e.g., Operation City Quest) will focus on different factors assessed in the protocol. These uses will help people with planning more efficiently by isolating characteristics based on customers' backgrounds.

\section{Conclusions}

Spatial navigation is one of the most indispensable skills for living organisms. A good navigator is typically associated with a well-rounded set of characteristics, such as a good memory, low spatial anxiety, and the use of allocentric strategies. Not being a good navigator, however, does not doom a person to ineffective navigation, because navigation is not always a one-person task. Collective navigation, by turning the entire group into essentially one well-rounded navigator, brings unlimited opportunities to solve navigation problems that would challenge each individual. The proposed prescriptive guideline for this scenario provides just one theoretical approach to solve the problem. More importantly, we used the guidelines as a starting point to re-evaluate the factors that are relevant to individual navigation, to initiate the assessment of factors that are unique to collective navigation, and to pave the way to look at spatial navigation in the social dimension. 


\section{References}

Astur, R. S., Ortiz, M. L., \& Sutherland, R. J. (1998). A characterization of performance by men and women in a virtual Morris water task:: A large and reliable sex difference. Behavioural brain research, 93(1-2), 185-190.

Boone, A. P., Gong, X., \& Hegarty, M. (2018). Sex differences in navigation strategy and efficiency. Memory $\&$ Cognition, (May), 1-14. https://doi.org/10.3758/s13421-018-0811-y

Chrastil, E. R., \& Warren, W. H. (2014). From Cognitive Maps to Cognitive Graphs. PLoS One, 9(11). https://doi.org/10.1371/journal.pone.0112544

Coluccia, E., \& Louse, G. (2004). Gender differences in spatial orientation: A review. Journal of environmental psychology, 24(3), 329-340.

Condon, D. M., Wilt, J., Cohen, C. A., Revelle, W., Hegarty, M., \& Uttal, D. H. (2015). Sense of direction: General factor saturation and associations with the Big-Five traits. Personality and Individual Differences, 86, 38-43. https://doi.org/10.1016/j.paid.2015.05.023

Elgar, M. A. (1989). Predator vigilance and group size in mammals and birds: a critical review of the empirical evidence. Biological Reviews, 64(1), 13-33.

Espinosa, M. P., \& Kovářík, J. (2015). Prosocial behavior and gender. Frontiers in behavioral neuroscience, 9, 88.

Goldberg, L. R. (1990). An alternative" description of personality": the big-five factor structure. Journal of personality and social psychology, 59(6), 1216.

Harris, H., Bailenson, J. N., \& Yee, N. (2009). The Evolution of Social Behavior. Presence, $18(6), 434-448$.

Harris, M. A., \& Wolbers, T. (2014). How age-related strategy switching deficits affect wayfinding in complex environments. Neurobiology of aging, 35(5), 1095-1102. 
Hegarty, M., Richardson, A., Montello, D., Lovelace, K., \& Subbiah, I. (2002).

Development of a self-report measure of environmental spatial ability. Intelligence, 30, 425-447. http://linkinghub.elsevier.com/retrieve/pii/S0160289602001162

Huff, D. L. (1964). Defining and estimating a trading area. The Journal of Marketing, $34-38$.

Lawson, B. D., \& Riecke, B. E. (2014). Perception of Body Motion.

Lawton, C. A. (1994). Gender differences in way-finding strategies: Relationship to spatial ability and spatial anxiety. Sex roles, 30(11-12), 765-779.

Lawton, C. A. (2001). Gender and Regional Differences in Spatial Referents Used in Direction Giving. Sex Roles, 44(5/6), 321-337. https://doi.org/10.1023/A:1010981616842

Lawton, C. A., \& Kallai, J. (2002). Gender differences in wayfinding strategies and anxiety about wayfinding: A cross-cultural comparison. Sex Roles, 47(9-10), 389-401. https://doi.org/10.1023/A:1021668724970

Lester, A. W., Moffat, S. D., Wiener, J. M., Barnes, C. A., \& Wolbers, T. (2017). The Aging Navigational System. Neuron, 95(5), 1019-1035. https://doi.org/10.1016/j.neuron.2017.06.037

Marchette, S. A., Bakker, A., \& Shelton, A. L. (2011). Cognitive Mappers to Creatures of Habit : Differential Engagement of Place and Response Learning Mechanisms Predicts Human Navigational Behavior. 31(43), 15264-15268. https://doi.org/10.1523/JNEUROSCI.3634-11.2011

Moffat, S. D., Hampson, E., \& Hatzipantelis, M. (1998). Navigation in a "virtual" maze: Sex differences and correlation with psychometric measures of spatial ability in humans. Evolution and Human Behavior, 19(2), 73-87.

Money, J., \& Alexander, D. (1966). Turner's syndrome: further demonstration of the presence of specific cognitional deficiencies. Journal of Medical Genetics, 3(1), 47. 
Money, J., Alexander, D., \& Walker, H. T. (1965). A standardized road-map test of direction sense: Manual. Johns Hopkins Press.

Padilla, L. M., Creem-regehr, S. H., \& Stefanucci, J. K. (2017). Sex differences in virtual navigation influenced by scale and navigation experience. Psychonomic bulletin $\&$ review, 582-590. https://doi.org/10.3758/s13423-016-1118-2

Pulliam, R. H. (1973). On the advantages of flocking. J. theor. Biol., 38.

Soutschek, A., Burke, C. J., Beharelle, A. R., Schreiber, R., Weber, S. C., Karipidis, I. I., Ten Velden, J., Weber, B., Haker, H., \& Kalenscher, T. (2017). The dopaminergic reward system underpins gender differences in social preferences. Nature Human Behaviour, 1(11), 819.

Spielberger, C. D., Gorsuch, R. L., Lushene, R., Vagg, P. R., \& Jacobs, G. A. (1983). Manual for the state-trait anxiety inventory Consulting Psychologists Press. Palo Alto, $C A$.

Talhelm, T., Zhang, X., Oishi, S., Shimin, C., Duan, D., Lan, X., \& Kitayama, S. (2014). Large-scale psychological differences within China explained by rice versus wheat agriculture. Science, $344(6184), 603-608$.

Weisberg, S. M., \& Newcombe, N. S. (2016). How do (some) people make a cognitive map? Routes, places, and working memory. Journal of Experimental Psychology: Learning, Memory, and Cognition, 42(5), 768.

Wiener, J. M., Condappa, O. D., Harris, M. A., \& Wolbers, T. (2013). Maladaptive Bias for Extrahippocampal Navigation Strategies in Aging Humans. The Journal of Neuroscience, 33(14), 6012-6017.

https://doi.org/10.1523/JNEUROSCI.0717-12.2013 